\title{
Clustered States as a New Paradigm of Condensed Matter Physics
}

\author{
G. Alvarez ${ }^{a}$ and E. Dagotto ${ }^{a}$

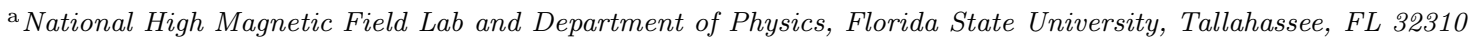

\begin{abstract}
We argue that several materials of much current interest in condensed matter physics share common phenomenological aspects that only very recent investigations are unveiling. The list includes colossal magnetoresistance manganites, high temperature superconducting cuprates, diluted magnetic semiconductors, and others. The common aspect is the relevance of intrinsic inhomogeneities in the form of "clustered states", as explained in the text.
\end{abstract}

Key words: Manganites, high temperature superconductors, diluted magnetic semiconductors, strongly correlated systems, computational physics.

PACS: 75.47.Lx, 74.25.Ha, 75.50.Pp

\section{Introduction}

In this paper it is argued that the recent trends unveiled in the context of manganites regarding the key role of inhomogeneities to explain the colossal magnetoresistance effect can be applied to a variety of other compounds as well. Notably, evidence is rapidly accumulating that underdoped high temperature superconductors are also inhomogeneous at the nanoscale, and theoretical approximations that assume homogeneous states are questionable. It is still unclear if the inhomogeneities have stripe features, as discussed extensively in previous years, or whether they correspond to more randomly shaped clusters, as assumed in the manganite context. Also the origin of these inhomogeneities is much debated. Computational simulations will play a key role in determining the properties of models for manganites and cuprates, since percolative clustered physics is difficult to handle with other methods. Even more recently, clustered states have also been discussed in a very unexpected context, the diluted magnetic semiconductors Mn-doped GaAs. These materials have considerable potential applications in spintronic devices and understanding their behavior may raise their current critical temperatures. In this publication, the three family of materials are briefly discussed and some key references provided such that the reader can appreciate the common trends between the many compounds. These quite unexpected similarities between apparently very different materials promises to lead to a global unified view, where clustered states become a new paradigm of condensed matter physics.

\section{Manganites}

Manganites are currently attracting considerable attention mainly due to the presence of the colossal magnetoresistance effect in magnetotrans- 
$\mathbf{T} \uparrow$

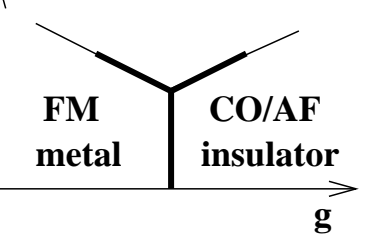

$\mathbf{T} \uparrow$
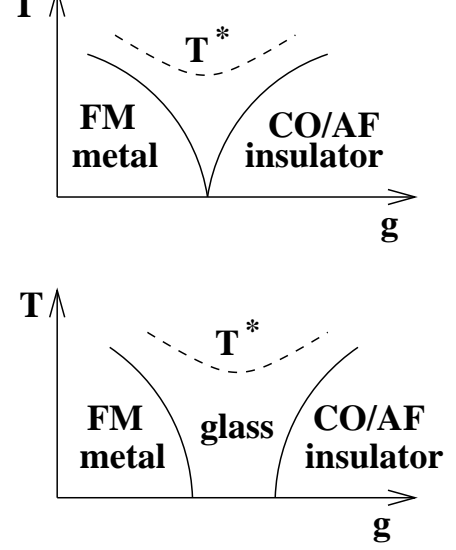

Fig. 1. Top General phase diagram of two competing phases in the absence of quenched-disorder (or when this disorder is very weak). Thick (thin) lines denote first (second) order transitions. $g$ is some parameter needed to change from one phase to the other. Middle With increasing disorder, the temperature range with first-order transitions separating the ordered states is reduced, and eventually for a fine-tuned value of the disorder the resulting phase diagram contains a quantum critical point. In this context, this should be a rare occurrence. Bottom In the limit of substantial quenched disorder, a window without any long-range order opens at low temperature between the ordered phases. This disordered state has glassy characteristics and it is composed of coexisting clusters of both phases. The size of the coexisting islands is regulated by the disorder strength and range, and by the proximity to the original first-order transition. For more details see Refs. 2, 3]. The new scale $T^{*}$, remnant of the clean-limit transition, is also shown.

port measurements [1, 2]. In addition, these materials have a complex phase diagram with a plethora of ordered phases, a characteristic of correlated electron systems. A variety of experimental and theoretical investigations have unveiled the inhomogeneous character of the states of relevance to explain the CMR phenomenon, with a competition between ferromagnetic and antiferromagnetic states that induces coexistence of clusters at the nanometer-scale [2].

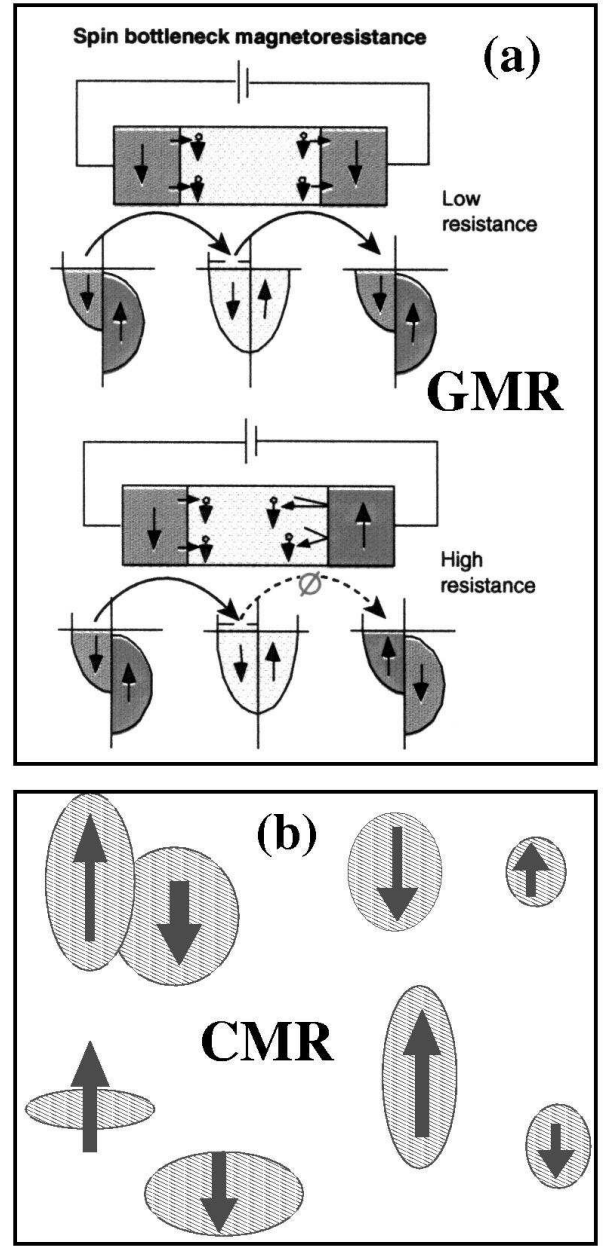

Fig. 2. Figure illustrating the analogies between CMR and GMR materials. (a) Schematic representation of a spin-valve effect. (Top) is made out of two ferromagnetic regions with aligned spins. In this case low resistance is achieved. (Bottom) consists of antiparallel ferromagnetic moments, and in this case the device has a high resistance. Reproduced from [5]. (b) Ferromagnetic clusters in the CMR materials. The arrows indicate orientation of magnetic moments (assuming Ising spins for simplicity). Transport occurs if moments are aligned, as in GMR. Possible coexisting insulating regions are not shown.

To understand this phenomenon, and its associated CMR effect, the first-order transitions that separate the metallic and insulating phases in the clean limit (i.e. without disorder) play a key role 1, 2]. The first-order character of the transition is caused by the different magnetic and charge orders of the competing states. The clean-limit phase di- 
agram is illustrated in Fig. 1 (upper panel). When quenched disorder is introduced in the coupling or density that is modified to change from one phase to the other, the temperature where the Néel and Curie temperatures meet is reduced in value and eventually collapses to zero as in Fig. 1 (middle panel). Further increasing the disorder strength, a spin glassy disordered region appears at low temperatures involving coexisting clusters (Fig. 1 (lower panel)). Simulations by Burgy et al. 3] have shown that the clustered state between the Curie temperature and the clean-limit critical temperature $\left(T^{*}\right)$, with preformed ferromagnetic regions of random orientations, has a huge magnetoresistance since small fields can easily align the moments of the ferromagnetic islands, leading to a percolative conductor in agreement with experiments 4]. This establishes qualitative similarities with "Giant MR" (GMR) multilayered materials, as sketched in Fig.2. The quenched disorder simply triggers the stabilization of the cluster formation, but phase competition is the main driving force of the mixed state. Elastic deformations may also play a key role in this context [6]. We refer the reader to the literature cited here, and references therein, for a more detailed view of this exciting area of investigations. The readers can also consult a list of "open problems" in the manganite context that has been recently provided [7].

\section{High temperature superconductors}

The results obtained for manganites are not crucially dependent on the nature of the two competing phases. The argumentation should be valid in the context of high temperature superconductors (HTS) as well, where an antiferromagnetic (AF) insulator (probably with stripes) competes with a $d$-wave superconductor (dSC). In the clean-limit, difficult to obtain with the standard chemical doping, the conjecture is that the two phases would have been separated by a line of first-order transitions as shown in Fig. 3. The inevitable disorder of the chemical doping procedure opens a window, and in this context the glassy state that separates the insulator from the superconductor is made up of small clusters of the two competing phases 3,8$]$ very different from the homogeneous exotic states proposed for that regime.

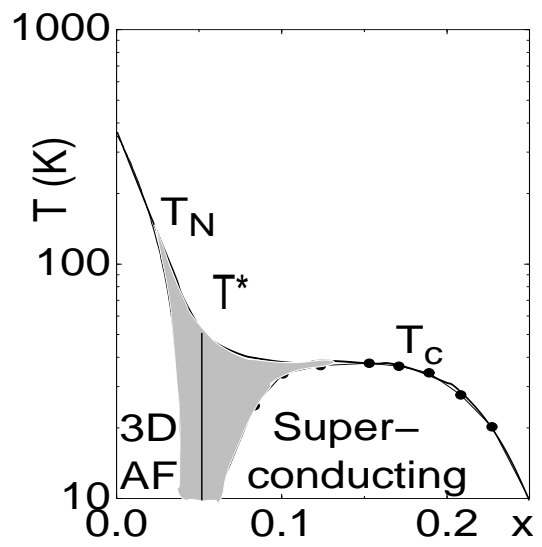

Fig. 3. Conjectured HTS phase diagram. The black lines should be the actual phase boundaries without disorder. The shaded region is conjectured to have metallic (SC) and insulating (AF) coexisting regions in the real materials.

First-order transitions AF-dSC have already appeared in electron doped materials [9], and they are also common in heavy-fermion and inorganic superconductor contexts.

Considering the general character of the discussion here, there should be an analog of the colossal effects in cuprates as well. In fact, colossal effects should be ubiquitous when ordered phases compete. A possible manifestation of this effect could be the "Giant Proximity Effect" recently discussed 10. with uncorrelated preformed SC clusters - in the sense that their phases in the order parameter are random - percolating under the influence of nearby superconducting materials and leading to a strong Josephson coupling across the originally non-superconducting sample.

These conjectured results for cuprates, where inhomogeneities play a key role, are in excellent agreement with the most exciting recent experimental developments in the high-Tc arena, namely the scanning tunneling microscopy experiments that unveiled the clustered nature of these materials at the nanoscale. 11 . 


\section{Diluted Magnetic Semiconductors}

Diluted magnetic semiconductors (DMS) are attracting much attention lately due to their potential for device applications in the growing field of spintronics. In particular, a large number of DMS studies have focused on III-V compounds where Mn doping in InAs and GaAs has been achieved using molecular beam epitaxy (MBE) techniques. The main result of recent experimental efforts is the discovery 12, 13, 14] of ferromagnetism at a Curie temperature $T_{\mathrm{C}} \sim 110 \mathrm{~K}$ in $\mathrm{Ga}_{1-x} \mathrm{Mn}_{x} \mathrm{As}$, with $\mathrm{Mn}$ concentrations $x$ up to $10 \%$. It is widely believed that this ferromagnetism is "carrier induced", with holes donated by Mn ions mediating a ferromagnetic interaction between the randomly localized $\mathrm{Mn}^{2+}$ spins. In practice, anti-site defects reduce the number of holes $n$ from its ideal value $n=x$, leading to a ratio $p=(n / x)$ substantially smaller than one. (a)

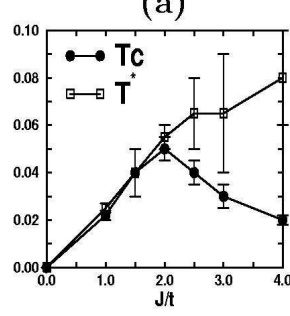

T4

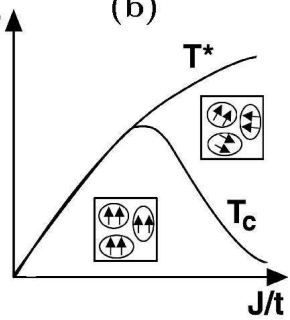

(c)

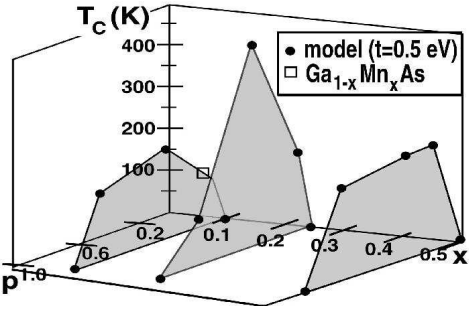

Fig. 4. (a) Schematic phase diagram varying $J / t$, at fixed $x$ and $p$. At large $J / t$, a broad scale $T^{*}$ corresponds to the formation of uncorrelated clusters, as indicated. $T_{\mathrm{C}}$ is the "true" transition temperature. At small $J / t$, those temperatures are similar. The optimal $J / t$ is intermediate between itinerant and localized regimes. (b) is the same as (a), but indicating the clustered states and moment orientations. (c) Numerically obtained $T_{\mathrm{C}}$ vs. $x$ and $p$, at $J / t=2.0$. Filled circles are from model Eq.(1) with $t=0.5$ $\mathrm{eV}$, while the open square corresponds to the experimental value for $\mathrm{Ga}_{1-x} \mathrm{Mn}_{x}$ As at $x \sim 0.1$. (Results from Ref. 15])

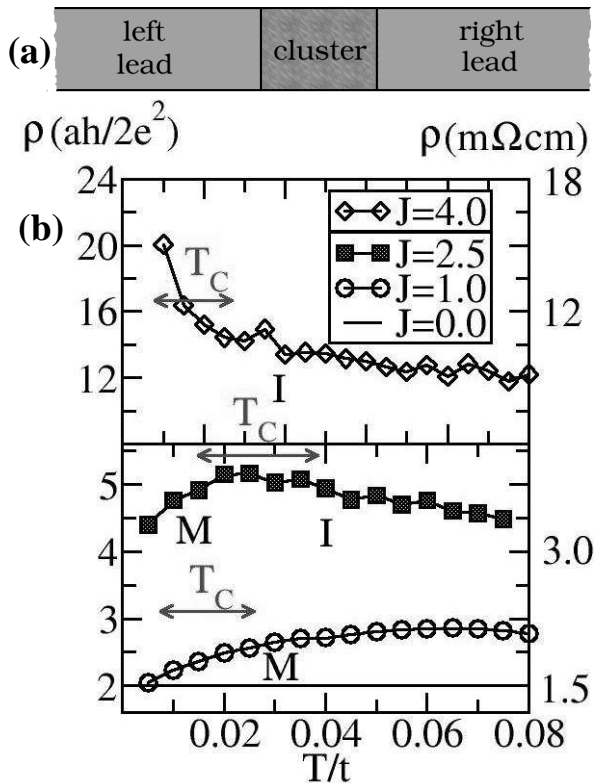

Fig. 5. (a) Geometry used for the calculation of the conductance. The interacting region (cluster) is connected by ideal contacts to semi-infinite ideal leads. (b) Dependence of the theoretically calculated resistivity, $\rho$, with temperature in three dimensions. Shown is $\rho=L / G$ vs. $T$ on $L^{3}=4^{3}$ lattices, 16 spins $(x=0.25)$, and 5 carriers $(p=0.3)$ for the $J / t$ 's indicated. An average over 20 disorder configurations has been performed in each case. Units are shown in two scales, $a h /\left(2 e^{2}\right)$ on the left and $\mathrm{m} \Omega \mathrm{cm}$ on the right, with $L=4$ and assuming $a=5.6 \AA$. The estimated critical temperatures are also shown (the arrows indicate the current accuracy of the estimations). (Results from Ref. 17])

In this section, generic Monte Carlo (MC) studies of a diluted Kondo lattice model for DMS are summarized. 15, 16, 17] Further information can be obtained from the cited references. The Hamiltonian is similar to those employed in the Mn-oxide context, namely

$$
\hat{H}=-t \sum_{<i j>, \sigma} \hat{c}_{i \sigma}^{\dagger} \hat{c}_{j \sigma}+J \sum_{I} \boldsymbol{S}_{I} \cdot \boldsymbol{\sigma}_{I},
$$

where $\hat{c}_{i \sigma}^{\dagger}$ creates a hole at site $i$ with spin $\sigma$, and the hole spin operator interacting antiferromagnetically with the localized $\mathrm{Mn}$-spin $\boldsymbol{S}_{I}$ is $\boldsymbol{\sigma}_{I}=\hat{c}_{I \alpha}^{\dagger} \boldsymbol{\sigma}_{\alpha, \beta} \hat{c}_{I \beta}$. The carrier can visit any site of the lattice. The interaction term is restricted to randomly selected sites, $I$, with a $S=5 / 2 \mathrm{Mn}$ moment. These spins are here considered classical with $\left|\boldsymbol{S}_{I}\right|=1$, as widely assumed, allowing 
for a MC simulation technically similarly as in manganites 1, 2]. Approximations include the neglect of on-site Coulomb repulsion $U$, valid at small $x$ and $p$ where double occupancy is unlikely even at $J / t=0$. In addition, Mn-oxides investigations 2. show that an intermediate or large $J / t$ plays a role analogous to a Hubbard $U / t$ at any $x$ [1]. At low $x$, the probability of nearest-neighbors (NN) Mn-spins is also low $(0.0625$ at $x=0.25)$, justifying the neglect of an anti-ferromagnetic (AF) Mn-Mn coupling. The hole motion is described by a oneband tight-binding model, while many bands may be necessary [18]. Despite this simplification, our model contains spins and holes in interaction and it is expected to capture the main qualitative aspects of carrier-induced ferromagnetism in DMS materials. Our effort builds upon previous important DMS theoretical studies [16, 19]. However, it differs from previous approaches in important qualitative aspects as explained in Ref. [15].

One of the most important observations in the study of Eq. (1) reported in [15], is the non-trivial dependence of $T_{\mathrm{C}}$ with coupling, $J / t$. For $J / t \rightarrow \infty$ and a Mn dilute system, the holes are trapped in Mn-sites, reducing drastically $T_{\mathrm{C}}$. Small FM clusters of spins are formed at a temperature scale $T^{*}$, but there is no correlation between them, leading to a global vanishing magnetization [16]. On the other hand, when $J / t=0$ the system is non-interacting. Since both in the $J / t \sim 0$ and $J / t=\infty$ limits $T_{\mathrm{C}}$ is suppressed, an optimal $J /\left.t\right|_{\text {opt }}$ must exist where $T_{\mathrm{C}}$ is maximized. This can be seen in Fig.4a-b. 15]

The $(x, p)$ dependence of $T_{\mathrm{C}}$ is shown in Fig. $4 \mathrm{c}$, assuming $t=0.3 \mathrm{eV}$. 15 In this figure, the best value of $T_{\mathrm{C}}$ achieved experimentally for GaMnAs is also indicated approximately. Note that increasing $x$ beyond the experimental value of 0.1 would significantly increase $T_{\mathrm{C}}$. At $J /\left.t\right|_{\text {opt }}$, the best value is $x \sim 0.25$, but room- $T$ ferromagnetism would be possible even with $x \sim 0.15$. An increase of $T_{\mathrm{C}}$ could also be achieved if the compensation could be decreased; in the optimal case the best value would be close to $p=0.5$. These predictions seem in agreement with experimental developments since very recently $\mathrm{Ga}_{1-x} \mathrm{Mn}_{x}$ As samples with $T_{\mathrm{C}}$ as high as $150 \mathrm{~K}$ were prepared, 20] a result believed to be caused by an enhanced free-hole density.

Dynamical and transport properties have also been calculated for the model presented here [17]. Of special importance is the conductance, $G$, which is calculated using the Kubo formula adapted to geometries usually employed in the context of mesoscopic systems. 21] The cluster is considered to be connected by ideal contacts to two semi-infinite ideal leads, as represented in Fig. 5a. In Fig. 5b the inverse of the conductance, which is a measure of the resistivity, is plotted for a three-dimensional lattice at weak, intermediate, and strong coupling, at fixed $x=0.25$ and $p=0.3$. For the weak coupling regime $(J / t=1.0)$ the system is weakly metallic at all temperatures. In the other limit of strong couplings, $1 / G$ - proportional to the resistivity $\rho-$ decreases with increasing temperature, indicating a clear insulating phase, as a result of the system being in a clustered state at the temperatures explored, 15, 16] with carriers localized near the Mn spins. At the important intermediate couplings emphasized in our effort, the system behaves like a dirty metal for $T<T_{\mathrm{C}}$, while for $T_{\mathrm{C}}<T<$ $T^{*}, 1 / G$ slightly decreases with increasing temperature, indicating that a soft metal to insulator transition takes place near $T_{\mathrm{C}}$. For $T>T^{*}$, where the system is paramagnetic, $1 / G$ is almost constant. Note that for strong enough $J / t, T_{\mathrm{C}} \rightarrow 0$ and therefore no metallic phase is present.

It is interesting to compare these numerical results with experiments. For $\mathrm{Ga}_{1-x} \mathrm{Mn}_{x} \mathrm{As}$, data similar to those found in our investigations have been reported. 12, 13, 14] The qualitative behavior of the resistivity in these samples agrees well with the theoretical results presented in Fig. 5b if intermediate couplings are considered.

The clustered state that forms just above $T_{\mathrm{C}}$ is a candidate to describe DMS materials since it explains both the resistivity maximum around $T_{\mathrm{C}}$, as well as the decrease in resistivity with increasing applied magnetic field. In addition, it provides an optimal $T_{\mathrm{C}}$. This clustered state could be unveiled using scanning tunneling microscopy techniques.

Recent experimental work on $(\mathrm{Ga}, \mathrm{Cr}) \mathrm{As}$ have revealed unusual magnetic properties which were associated with the random magnetism of the alloy. The authors of Ref. 22 explained their results using a distributed magnetic polaron model, that resembles the clustered-state ideas discussed here and in Refs. [15, 23. The present simulations un- 
veils a previously unknown similarity between DMS materials and transition metal oxides, since both have regimes where clustered states dominate. This analogy deserves further work.

\section{Conclusions}

The information provided here is indicative of the key role that inhomogeneous clustered states play in many materials. Their existence leads to quite nontrivial results. The colossal magnetoresistance effect appears to be linked to their presence. Giant proximity effects in cuprates may have a similar origin. The physics of important materials for applications such as diluted magnetic semiconductors may share a similar phenomenology, including a large magnetoresistance. This field is at an early stage in its development, which will need a coordinated effort between theorists - mainly those with expertise in simulations - and experimentalists. The study of clustered states is rapidly developing into one of the most active areas of present day condensed matter physics.

Work supported by NSF grant DMR-0122523.

References

[1] A. Moreo, S. Yunoki and E. Dagotto, Science 283, 2034 (1999); E. Dagotto, T. Hotta, and A. Moreo, Phys. Reports 344, 1 (2001); Y. Tokura, editor, Colossal Magnetoresistive Oxides, Gordon and Breach, 2000; and others.

[2] E. Dagotto, Nanoscale Phase Separation and Colossal Magnetoresistance, Springer-Verlag, Berlin, 2002.

[3] J. Burgy, M. Mayr, V. Martin-Mayor, A. Moreo, E. Dagotto, Phys. Rev. Lett. 87, 277202 (2001).

[4] M. Uehara, S. Mori, C. H. Chen, and S.-W. Cheong, Nature 399, 560 (1999); M. Fäth, S. Freisem, A. A. Menovsky, Y. Tomioka, J. Aarts, and J. A. Mydosh, Science 285, 1540 (1999).
[5] G. A. Prinz, Science 282, 1660 (1998). See also G. A. Prinz, Phys. Today, April 1995, p. 58.

[6] K. H. Ahn et al., cond-mat/0207224 A. R. Bishop et al. cond-mat/0304198 Jian-Xin Zhu et al., cond-mat/0304668 J. Burgy et al., in preparation.

[7] E. Dagotto, Open Questions in CMR Manganites, Relevance of Clustered States, and Analogies with Other Compounds, cond-mat/0302550 See also Aliaga et al., cond-mat/0303513

[8] An alternative is to have stripes, as discussed in V. Emery and S. Kivelson, Physica C 209, 597 (1993). See also S. Caprara, C. Di Castro, M. Grilli, Proceedings of the XXII International Conference on Low Temperature Physics August 4-11, 1999, Espoo, Physica B 284-288, 983 (2000) and references therein.

[9] N. Harima et al., cond-mat/0103519

[10] R. S. Decca et al., Phys. Rev. Lett. 85, 3708 (2000). See also I. Bozovic, preprint submitted to Science, and private communication.

[11] K. M. Lang et al., Nature 415413 (2002) and references therein.

[12] H. Ohno, Science, 281951 (1998); H. Ohno, J. Magn. Magn. Mater., 200, 110 (1999).

[13] S. Katsumoto, et al., Mater. Sci. and Eng. B 84, 88 (2001).

[14] S. J. Potashnik et al., Appl. Phys. Lett. 79, 1495, (2001).

[15] G. Alvarez, M. Mayr and E. Dagotto, Phys. Rev. Lett. 89, 277202 (2002).

[16] M. Mayr, G. Alvarez, and E. Dagotto, Phys. Rev. B 65, 241202 (2002)

[17] G. Alvarez and E. Dagotto, cond-mat/0303350 To appear in Phys. Rev. B.

[18] J. Schliemann and A. H. MacDonald, Phys. Rev. Lett. 88, 137201 (2002); J. Schliemann et al., Phys. Rev. B64, 165201 (2001); Appl. Phys. Lett. 78, 1550 (2001).

[19] T. Dietl, cond-mat/0201282 Mona Berciu and R. N. Bhatt, Phys. Rev. Lett. 87, 107203 (2001). A. Kaminski and S. Das Sarma, Phys. Rev. Lett. 88247202 (2002). A. Chattopadhyay et al., Phys. Rev. Lett. 87, 227202 (2001). M. J. Calderón et al., cond-mat/0203404 
[20] K. C. Ku et al., cond-mat/0210426 (2002).

[21] J. A. Verges, Comp. Phys. Commun. 118, 71 (1999).

[22] A. Dakhama et al. Phys. Rev. B 67, 115204 (2003).

[23] C. Timm et al., Phys. Rev. Lett. 89, 137201 (2002). 